\title{
The Conditions for Se-Enzymes, NO, cGMP and LDL to be Formed and How They are Connected with Each Other
}

\author{
Birgit Elisabeth Swenson ${ }^{*}$ \\ Chalmers Technical University, Molndal 43136, Sweden
}

Received: January 09, 2014 / Accepted: January 28, 2014 / Published: March 25, 2014.

\begin{abstract}
Transportation of energy and substances is fundamental for the conditions of life. The energy from metabolised food is yielded at the reduction of oxygen by the assistance of Se-enzymes. Activated Se-enzymes contain an electron conducting Se-link with elemental Se bounded to Se-cys (Se-cystein) that prevents electron transferring (autoxidable) agents, as arginine, from oxidations. NO is formed from e.g. arginine during the passive state of a Se-enzyme. With NO, instead of $\mathrm{O}_{2}$, bounded to a hem group, destroying oxidations are avoided. This is the role of NO. A nerve signal is activated by the GTP (guanosine triphosphate)-induced triggered transformation of cGMP (cyclic guanosine monophosphate) into GMP, closing the sodium channels. When GTP is consumed, the opposite reaction takes place. The direct influence of the GMP/cGMP quote on a nerve signal makes GMP/cGMP equal to EDHF (endothelium-derived hyperpolarizing factor). Part of the energy from food is stored and transported in the form of acetyl groups, building up many important molecules of which LDL (low density lipoprotein) is one, containing cholesteryl esters. These are brought in to the cell, decomposed to acetyl groups generating hydrogen, H, making LDL to an essential substance. High levels are connected to impaired energy yielding reactions, perhaps related to low levels of some substances, especially to one or both forms of Se. The necessity of a Se-link and NO induced protection against oxidations by reducible oxygen can no longer be neglected.
\end{abstract}

Key words: Se-enzymes, Se-toxicity, formation of NO, formation of cGMP, EDHF, cholesteryl esters from LDL.

\section{Introduction}

Do you like to play with balls?

It is an extremely fast and complicated process how the sight of a flying ball starts a system of reactions that leads to nerve signals directing the contraction of muscles in, hopefully, the right way for treating the ball as you meant to. No one knows how many reactions and substances that are involved in that process.

Se-enzymes, LDL (low density lipoprotein), NO and cGMP (cyclic guanosine monophosphate) are four of them. However, their reactions are mainly unknown.

${ }^{*}$ Corresponding author: Birgit Elisabeth Swenson, Diploma Engineer, research field: chemical reactions in medical research. E-mail: birgit.swenson@telia.com.
The energy from food is yielded after degradation to hydrogen $(\mathrm{H}$, containing one proton and one electron) and reduction of $\mathrm{O}_{2}$ into $\mathrm{H}_{2} \mathrm{O}$ which can take place in two or three steps with $\mathrm{O}_{2}^{-}$and $\mathrm{H}_{2} \mathrm{O}_{2}$ as important intermediates. Everyone knows that our life is dependent on a consumption of $\mathrm{O}_{2}$. Nature invented the first fuel cell.

As soon as the production of $\mathrm{O}_{2}$ on the earth had started from photosynthetic processes, the evolution could take a huge step forward by using $\mathrm{O}_{2}$ in respiration with a much larger yield of energy compared to only fermentation. That explains why $\mathrm{O}_{2}$ is of utmost importance.

However, not many are aware of $\mathrm{O}_{2}$ as the only but most harmful radical. $\mathrm{O}_{2}$ can not donate electrons and consequently not react with itself which is the case for 
$\mathrm{H}_{2} \mathrm{O}_{2}$, OH and $\mathrm{O}_{2}^{-}$forming $\mathrm{O}_{2}$ by auto-reactions. The high reactivity of $\mathrm{O}_{2}$ makes it necessary to have enzyme protected reactions to avoid destroying oxidations. Otherwise, organisms could not utilize energy in a proper way to maintain the processes of life. Many steps are known in energy yielding processes with reducible oxygen but important steps are still missing, especially when the energy is used for nerve induced muscle activities.

However, from lots of experiments, certain conclusions can actually be drawn to put some light over such processes.

In this paper, the author will describe how Se-enzymes, NO, cGMP and LDL are related to the active and inactive states and under which conditions they are formed and involved in the $\mathrm{O}_{2}$ protection. They are most important substances which roles and reactions have not been properly understood.

\section{Activation of Se-Enzymes}

Protection against hazardous oxidations had to be found by the evolution before $\mathrm{O}_{2}$ could be utilized. It was found many million years ago. Minerals in the rocks were the only solid source of elements. Nature found sulphur minerals as pyrite, $\mathrm{FeS}_{2}$ that solved the problem with $\mathrm{O}_{2}$. They contain small quantities of $\mathrm{Se}$ (selenium). All the three elements $\mathrm{Fe}, \mathrm{S}$ and Se were used in the reduction of reducible oxygen to gain energy without destroying oxidations. However, surprisingly it has so far been unknown though Sirén already in 1964 postulated that the photoreceptors of the eyes contain elemental Se which transduces photons to electric signals [1].

When exposed to only red or pink light, rodents put on weight and after some time, died. Rodents lost their tails, ears and finally life when exposed to only one colour of the light, especially the orange, red or pink light [2].

The absorption curve for selenium photo cells and the human eye are almost identical and the absorption max corresponds to the blue green light why red light is reflected.

No energy is yielded for the processes of life without an activation of Se despite how much food an organism has consumed.

Exhausted horses became calm much faster in a blue-green stable compared to a red-orange [2].

It is well documented that Se-enzymes contain Se-cys (Se-cystein) and that the right reaction of a Se-enzyme is fundamental for the function of many systems.

It is a general but wrong opinion that Se-enzymes contain only Se-cys [3, 4].

Experiments have shown that Se-enzymes are activated by $\operatorname{Se}(0)$ from e.g., selenite, methylseleninate, GSSeSG (seleno diglutathione) [5]. $\mathrm{Se}(0)$ easily binds to $\mathrm{Se}(-\mathrm{II})$ in an enzyme, forming an electron conducting Se-link, -Se-Se', to reducible oxygen. A protection against oxidations of the electron transferring substances (autoxidable agents) was found which prevents a contact between $\mathrm{O}_{2}$ and the autoxidable agent $[3,4]$.

What could be expected, there is not a permanent support of energy for the activation of Se-links, the transference of electrons. When it is ended, $\operatorname{Se}(0)$ is oxidized into soluble $\mathrm{SeO}_{2}$ starting the inactive period.

Consequently, Se-enzymes are only found to contain Se-cys during the inactive period. It is not possible to analyze them during an active period [5].

Possibly, the reactions of Se-enzymes have not been understood because of the misunderstanding that $\mathrm{Se}(0)$ can be reduced to $\mathrm{Se}(-\mathrm{II})$ [3]. It is a huge difference between the toxicity of selenite and Se(-II)-compounds [5]. Consequently, selenite cannot be transformed into $\mathrm{Se}(-\mathrm{II})$. It has never been proofed. It can never take place in an aerobic organism as oxygen is so much easier reduced.

The toxicity of selenite and other $\mathrm{Se}(0)$-forming compounds depends on the formation of links with thiols, impairing the redox reactions of thiols $[5,6]$. Consequently, mercapto compounds may prevent the toxicity of selenite. After ingestion of selenite in toxic 
doses, a support of dimercaprol, containing two SH-groups, indicated that it eliminated the toxic effect [7].

Besides, there is a lack of insight about the role of $\mathrm{H}_{2} \mathrm{O}_{2}$. It is a fact that $\mathrm{H}_{2} \mathrm{O}_{2}$ is formed or reduced in the reactions of Se-enzymes e.g. thioredoxin reductase, iodinases (ID I-III), xanthindehydrogenas, Gpx (glutathionperoxidas).

NOS (NO synthase) dependent production of superoxide and $\mathrm{H}_{2} \mathrm{O}_{2}$ from the endothelium has been reported [8]. NOS appears to be one of the sources of the acetylcholine (ACh)-induced $\mathrm{H}_{2} \mathrm{O}_{2}$ production from the endothelium, since ACh-induced $\mathrm{H}_{2} \mathrm{O}_{2}$ production was markedly reduced when NOS in blood vessels was inactivated [9].

It can surely be assumed that all enzymes yielding energy by reducing oxygen, stored and transported in ATP or GTP, are Se-enzymes.

The Se-link-dependent formation and reduction of $\mathrm{H}_{2} \mathrm{O}_{2}$ are undoubtedly essential reactions for the support and transportation of energy for the processes of life [3-6].

\section{Are Se-Enzymes Related to NO?}

Arginine (arg) is a necessary substance for the function of many organs. It is well known that arginine can decompose to ornithine or citrulline and NO by NOS but be rebuilt by the urea cycle. From arginine, creatine is formed which has the same reactive group as arginine. Under normal conditions, about half of the Se content in the organism is found in the skeletal muscles [10]. It has been observed a positive correlation between physical exercise and NO formation, which was higher in athletic subjects than in nonathletic [11]. The generation of energy is higher in athletes, and thus the reaction of creatine and the formation of NO $[3,4]$.

However, so far, no one has been able to explain why NOS at one time catalyses the ACh induced $\mathrm{H}_{2} \mathrm{O}_{2}$ production and at another NO. Which are the conditions when NO is formed and which when $\mathrm{H}_{2} \mathrm{O}_{2}$ is produced?
NO is a product when arginine is oxidized to citrulline by $\mathrm{O}_{2}$ under the support of NADPH (nicotinamide adenine dinucleotide phosphate, reduced form) and NOS. NO may probably be formed also from other autoxidable agents containing $\mathrm{N}$.

Obviously, there is no protection against oxidation. This means that Se-links are deactivated.

NO can only be produced when Se-links are inactive and $\mathrm{H}_{2} \mathrm{O}_{2}$ or $\mathrm{H}_{2} \mathrm{O}$ when they are active.

Ornithine, on the other hand, is surely produced without the existence of a Se-enzyme and a H-transport.

Besides Se, Nature utilizes the other elements in $\mathrm{FeS}_{2}$. Many proteins contain $\mathrm{S}$, reacting in redox reactions forming $\mathrm{S}$-links and $\mathrm{Fe}^{2+}$ is bounded to hem structures. It is also known that NO binds to the hem part of the cytochromoxidase occupying the oxygen binding site, thus allowing NO to regulate mitochondrial affinity for oxygen [12].

NO binds firmly to $\mathrm{Fe}^{2+}$ forming a brown red compound. This is done with $\mathrm{Fe}^{2+}$ in hem structures, frequent in many enzymes, preventing $\mathrm{O}_{2}$ from binding to them. Further oxidations are avoided.

However, NO affects cell metabolism. Endogenous NO inhibits mitochondrial respiration and ATP (adenosine triphosphate) synthesis. This is a consequence of inhibition of cytochromoxidase [12].

No energy is generated, why the process represents an inactive state. NO gives the protection against destructive oxidations during the passive phase. This phase is fundamental for the processes of life to get systems prepared, with the right substances at the right places for a new active phase. For example, kinase enzymes replace phosphate groups to where they are available for GDP (guanosine diphosphate) or ADP (adenosine diphosphate) to form GTP (guanosine triphosphate) or ATP during the active phase, citrulline is reformed to arginine. The active and passive phases must regularly replace each other.

$\mathrm{NO}$ is oxidized to soluble nitrite or nitrate and $\mathrm{O}_{2}$ can bind to $\mathrm{Fe}^{2+}$ hem starting a new active phase. Thus, 
in aerobic organisms, $\mathrm{NO}$ is the protection from $\mathrm{O}_{2}$ oxidations when the Se-link is in a passive phase.

It surely could be verified by experiments that the high level of NO in exhaled air from asthmatics or premature and term infants will decrease if the functions of Se can be increased.

However, the authors do not need experiments to be sure that a higher level of NO related to health problems will be reduced with a reduction of the health problems if they are correlated to impaired functions of Se.

You can never understand the complete role of NO, involving how and why it is produced, if you do not realize that protection against destructive oxidations is made by the Se-link during an active phase and NO during a passive.

\section{Is cGMP Dependant on NO?}

NO activates cytosolic guanylatcyclase. As a result, cyclic cGMP is increased and leads to vascular smooth muscle relaxation [13].

NO activates cGMP synthesis and increases inhibition of PDE (phosphodiesterase), which degrades cAMP [14] and cGMP [15].

It is well known that when NO binds to $\mathrm{Fe}^{2+}$ in the hem structure of guanylatcyclase, the cyclic form of GMP, cGMP, is promoted.

Stryer has described the steps involved in the cleavage of cGMP in the retina, starting with a light signal. When retinal absorbs a photon, opsin, containing phosphate groups, is activated, causing a triggered rodopsin induced activation of $\mathrm{T}$ (transducin) in which GTP is exchanged for GDP.

The $\alpha$-unit in T with GTP, T $\alpha$ GTP, binds to the $\gamma$-unit of a PDE activating it to insert a water molecule into cGMP in a highly triggered reaction. When cGMP is hydrolyzed into 5 GMP, the sodium channels close spontaneously and the light-induced hyper polarization is passed along the membrane to the synaptic terminal where the nerve impulse arises [15]. The active phase is ended.
The channels do not remain closed which means that cGMP is reproduced from $\mathrm{H}^{+}$-activated $5^{\circ} \mathrm{GMP}$, during the passive phase.

However, some questions have to be answered:

(1) From where does the energy come for the production of GTP?

(2) Is the formation of cGMP and cAMP connected to $\mathrm{NO}$ ?

An activation process must start with a generation of energy in which guanylatcyclase is involved as NO binds to the hem structure of guanylatcyclase. For the oxidation of $\mathrm{NO}, \mathrm{O}_{2}$ is needed. $\mathrm{NO}$ is undoubtedly replaced by $\mathrm{O}_{2}$, as is the case for cytochromoxidase [12]. This means that $\mathrm{O}_{2}$ binds to the hem structure of guanylatcyclase and energy is generated by ACh-induced $\mathrm{H}$ reducing $\mathrm{O}_{2}$ by the assistance of NOS, arginine and an Se-link, activated by the same light as rodopsin.

Stored energy in GTP is used for the reaction of cGMP into GMP, Fig. 1 [16]. Without GTP, the reverse reaction spontaneously takes place.

Thus, NO and cGMP represent a passive phase and can both be coupled to guanylatcyclase and NOS.

The toxins produced by the bacteria that cause cholera and whooping cough (pertussis) are enzymes whose actions result in a deactivation of an arginine residue of $\mathrm{G}$ proteins. The pathology of both diseases results from defective regulation of adenylate cyclase and overproduction of cAMP [17].

No energy is generated when arginine is deactivated. The ring opening of cAMP cannot take place.

As Stryer has described, nerve signals are depending on the GMP/cGMP quote. A formation of GTP from ACh-induced $\mathrm{H}_{2} \mathrm{O}_{2}$ production by the assistance of arginine and Se enzymes are surely involved. Several factors give evidence for cAMP and AMP to have a similar role, important directing substances for hormone systems depending on ATP or GTP [15].

The nature of EDHF (endothelium-derived hyperpolarizing factor), causing smooth muscle membrane 

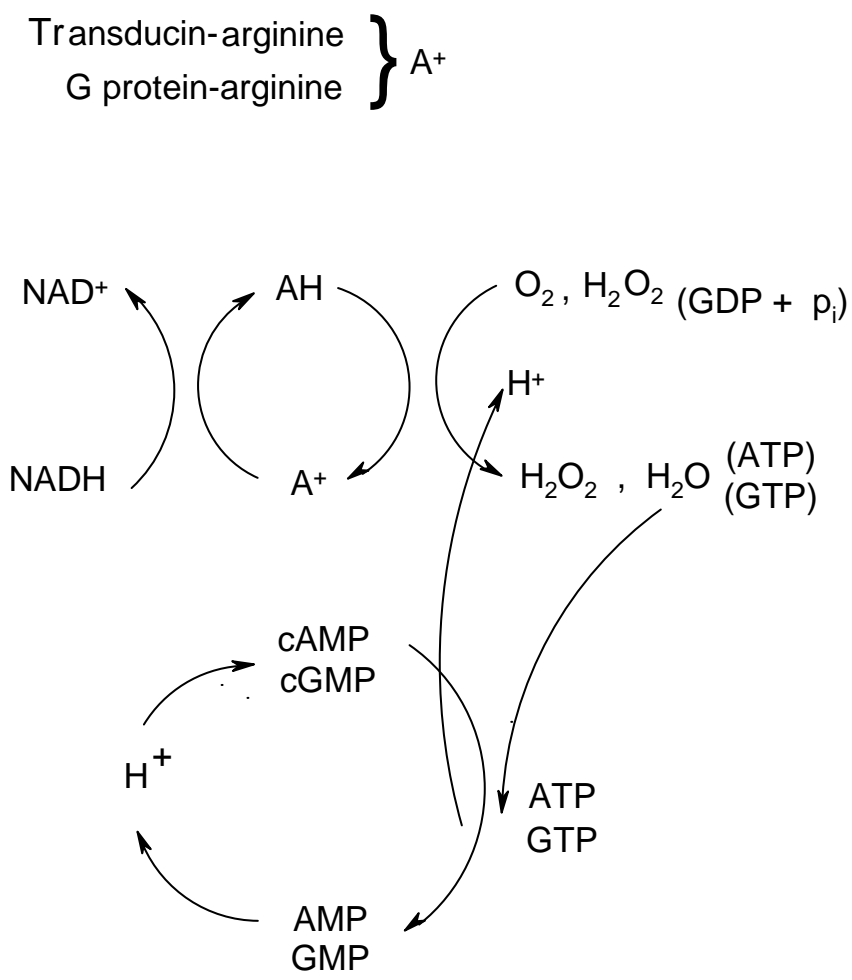

Fig. 1 The $G$ protein or transducin catalyzed generation of ATP (GTP) involved in the loop ATP-cAMP-AMP (GTP-cGMP-GMP) which regulates nerve and hormone signals.

hyper polarization followed by vasodilatation, is unknown. Proposals are made that NO by itself can be EDHF, but even in the presence of an NO scavenger, ACh still causes relaxation and hyper polarization in small arteries [13].

It has been suggested that $\mathrm{H}_{2} \mathrm{O}_{2}$ derived from endothelial NOS is an EDHF. EDHF-mediated relaxation and hyper polarization in response to $\mathrm{ACh}$ were markedly attenuated in small mesenteric arteries from inactivated NOS in mice [9].

Naturally, but in an inactive NOS system, the formation or reaction of many substances is low.

The possible role of cytochrome P450-derived $\mathrm{H}_{2} \mathrm{O}_{2}$ as an EDHF in coronary arteries of rats [18] and pigs [19] were examined with negative results.

Injected cGMP into the outer segment of intact rod cells, quickly reduced the potential difference across the plasma membrane. Not only that, the injected nucleotide sharply increased the delay between the arrival of a light pulse and the hyper polarization of the membrane [15].
cGMP is the only molecule that has shown an ability to directly regulate the sodium channels. When cGMP is hydrolyzed into 5 GMP, the sodium channels close spontaneously and the light-induced hyper polarization activates a nerve impulse. From what is known about cGMP and 5 GMP in the systems in which arginine and NO are active, the reaction of cGMP into GMP or the reverse, the GMP/cGMP quote, is the natural and clear choice, identical to EDHF. When it is $>>1$, the sodium channels close giving rise to a nerve impulse. When it is $<<1$, the sodium channels are open and a vasodilatation take place.

In a well regulated functional system, cGMP and 5 GMP are constantly replacing each other.

By the formation of NO, a passive state starts. GMP is changed into cGMP.

NO and cGMP are not produced in the same reaction, However, cGMP is a consequence of an NO-production since GTP is no longer available. That is why they are connected with each other. 


\section{Is LDL a Bad Cholesterol?}

Already in the middle of the last century, it was known that acetylcholine had to be quickly decomposed. If not, a consequence of an affection of pesticides, severe damage was noted, described in the book "Silent Spring" by Rachel Carson.

However, nor Rachel Carson or any one else could explain why it was so important with a quick decomposition.

Frequent reports of NOS-induced $\mathrm{H}_{2} \mathrm{O}_{2}$ from ACh give proof of a generation of energy in these systems. Like the acetyl groups from other sources in acetyl-CoA, ACh reacts producing free $\mathrm{H}^{-} \mathrm{e}^{-}$and $\mathrm{H}^{+}$. The acetyl group is the only consumed part of ACh as choline is not degraded but reutilized.

No one seems to have realized that the acetyl group in acetylcholine is the fuel for the function of nerves and other systems [16] depending on Se-enzymes of which NOS appears to be one, protecting arginine from oxidation $[3,4]$.

The storage and transportation of acetyl groups are consequently of great importance. That is why the acetyl group is a part of many molecules, Fig. 2 [16]. That is why it is of great importance to know how these molecules react.

However, not even the researchers at the big company Pfizer were aware of that.

They, like most people, regard LDL as the "bad cholesterol" causing heart diseases and by that reason intended to find a substance which almost eliminated the production of LDL. HDL (high density lipo protein) contains an enzyme which regulates the formation of LDL. By attacking this enzyme they reached their goal, confirmed by experiments. This resulted in a huge elevation of the value of the company. Only two days later, the catastrophe was a fact. Studies with humans showed that heart problems got worse and that actually $60 \%$ more people died.

Jonah Lehrer has written about that and how one billion dollar was spoiled during decades of research in order to develop a new drug, Torcetrapib, against LDL-induced high levels of cholesterol [20]. Besides, 90 million dollars were spent in the construction of a new factory.

However, it did not prevent the researchers at Pfizer to spoil another billion dollar in an attempt to find a drug against $\mathrm{AD}$ (Alzheimers desease). It failed by the same reason-lack of knowledge how ACh reacts and how it is involved in $\mathrm{AD}$ [6].

Cholesterol is a compound in the structure of membranes and many hormones are produced from it. HDL contains an enzyme that catalyzes the formation of cholesteryl esters from fatty acids in lecithin and cholesterol from lipo proteins in the plasma. LDL has a high content of cholesteryl esters which by receptors on the cell surfaces are brought into cells where they are hydrolyzed into cholesterol and fatty acids. Ahterosclerosis is related to high levels of cholesterol in the blood and especially to high levels of LDL

\section{Fats} Carbohydrates Proteins Ethanol

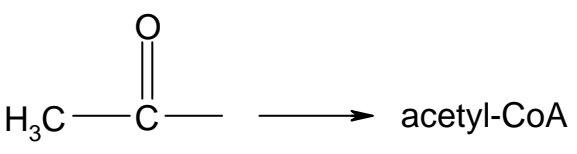

acetyl group

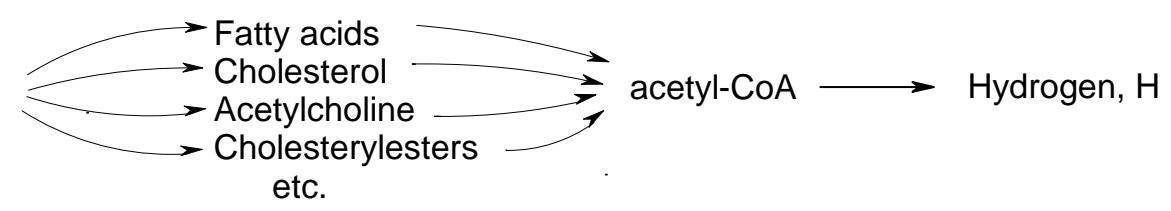

Fig. 2 The involvement of the acetyl group in many organic substances important to the processes of life. 
cholesterol whereas there is a negative correlation between high levels of HDL and atherosclerosis.

Cholesteryl esters serve for a transportation of acetyl groups. Many studies have shown that after small daily doses of ethanol the level of HDL is raised. HDL produces cholesteryl esters why a high level of HDL is combined with a high consumption of acetyl groups. On the contrary, a high level of LDL means that acetyl groups are poorly utilized. That can be explained by an impaired Se-induced reduction of reducible oxygen. The fatal consequence is a too low generation of energy which is the fundamental cause to health problems. However, if LDL is almost eliminated from a system, the generation of energy becomes too low to support the processes of life.

LDL is not a bad but an essential substance which is dependant on Se-enzymes.

\section{Summary: Important Steps for the Generation of Energy}

(1) An electron is excited and an Se-link is activated by blue green light or the corresponding energy.

(2) Autoxidable agents, such as arginine, transfer ACh-induced $\mathrm{H}$ into $\mathrm{O}_{2}$, reducing it by the assistance of a Se-link. Formed $\mathrm{H}_{2} \mathrm{O}_{2}$ is further reduced, by a Se-dependant Gpx.

(3) The generated energy is stored and transported in GTP or ATP and utilised in other processes such as the TaGTP-induced hydrolysis of cGMP into 5 GMP by PDE in the retina (Fig. 1 [15]).

(4) The consequence of the 5 GMP formation is that the sodium channels close giving rise to a nerve impulse.

(5) Se-links are only activated as long as energy is available - as in a light sensitive photoconductor. Without an activation, $\mathrm{Se}(0)$ is oxidized into $\mathrm{SeO}_{2}$, leaving Se-cys.

(6) Energy can no longer be generated. During this passive state, Se-enzymes contain only Se-cys.

(7) The autoxidable agent next to $\mathrm{O}_{2}$, no longer protected by an Se-link, is oxidized of which some of them produce NO e.g., arg, creatine and probably dopamine (DA) and others with an aminogroup. Arginine is oxidized into citrulline and DA into 3,4-DOPAC (dihydroxyphenylacetic acid).

(8) NO, instead of $\mathrm{O}_{2}$ binds to $\mathrm{Fe}^{2+}$ in hem structures of e.g., guanylatcyclase. Further oxidations are inhibited.

(9) As GTP is no longer produced, cGMP cannot be hydrolyzed into 5 GMP why the opposite reaction takes place. cGMP is reformed from 5 GMP by using protons, no longer utilized in a reduction process of $\mathrm{O}_{2}$ (Fig. 1) which is why NO-binding guanylatcyclase is regarded as an activator of the reaction.

(10) Phosphate groups from degraded GTP are replaced by kinases to proteins, as opsin in the retina, to be available for the next active phase when GDP will bind a phosphate group to form GTP. Consequently, GTP must be formed in the outer membrane of the retina and not in a mitochondrion.

(11) $\mathrm{NO}$ is oxidized by $\mathrm{O}_{2}$ to soluble nitrite or nitrate, leaving the $\mathrm{Fe}^{2+}$-hem in e.g. guanylatcyclase. $\mathrm{O}_{2}$ is exchanged for NO to be available when a new energy yielding cycle starts.

(12) Oxidized $\mathrm{Se}\left(\mathrm{SeO}_{3}{ }^{2-}\right)$ is reduced by vitamin $\mathrm{C}$ or $\mathrm{S}(-\mathrm{II})$ to $\mathrm{Se}(0)$ which binds to Se-cys in an Se-enzyme forming an Se-link which can be activated.

It can no longer be questioned which important roles Se-enzymes play in the protection against reactive oxygen.

The necessary need of energy for the processes of life has to be much more considered than it is today.

To utilize the $\mathrm{O}_{2}$ and NO-binding capacity of $\mathrm{Fe}^{2+}$ in life dependent energy yielding processes together with the pulse activated electron conducting Se-link from the same source, $\mathrm{FeS}_{2}$, is a brilliant solution of Nature.

\section{References}

[1] Siren, M. J. Is Selenium Involved in the Excitation Mechanism of Photoreceptors? The LKB Instrumental J. 1964, 11(3), 37-44. 
[2] Ryberg, K. Livingcolours. ICA-Company, Västerås, Sweden, 1999.

[3] Nyberg-Swenson, B. E. The Selenium Link: The Missing Link in Our Understanding of Biochemical Trigger Reactions? Medical Hypothesis. 1999, 52(2), 125-131.

[4] Nyberg-Swenson, B. E. Is Molecular Oxygen, $\mathrm{O}_{2}$, the Reactive Radical behind Oxidations of Aut Oxidable Agents to which the Bases of DNA belong? Medical Hypothesis 2002, 58(3), 203-212.

[5] Swenson, B. E. Injections of Substances inducing Apoptosis to Cancer Cells but Stimulation of Normal Cells may Prevent Cancer. Journal of Chemistry and Chemical Engineering 2011, 5(5), 393-403.

[6] Swenson, B. E. A Method and a Surface Treatment Agent for Preventing Biofouling on Surfaces under Water. Journal of Chemistry and Chemical Engineering 2012, 6(3), 227-232.

[7] Nuttail, K. L. Evaluating Selenium Poisoning. Ann Clin Lab Sci. 2006, 36(4), 409-420.

[8] Cosentino, F. Tetrahydrobiopterin Alters Superoxide and Nitric Oxide Release in Prehypertensive Rats. J Clin Invest. 1998, 101, 1530-1537.

[9] Matoba, T. Hydrogen Peroxide is an Endothelium-Derived Hyperpolarizing Factor in Mice. $J$. Clin Invest. 2000, 106(12), 1521-1530.

[10] Oster, O.; Schmiedel, G.; Prellwitz, W. The Organ Distribution of Selenium in German Adults. Biol. Trace Elem. Res. 1988, 15, 23-45.

[11] Jungersten, L. Both Physical Fitness and Acute Exercise Regulate Nitric Oxide Formation in Healthy Humans. $J$. Appl. Physiol. 1997, 82(3), 760-764.
[12] Fleming, I.; Busse, R. NO: Primary EDRF. J. Mol. Cell Cardiol. 1999, 31, 5-14.

[13] Stankevičius, E.; Kèvelaitis, E.; Vainorius, E.; Simonsen, U. Role of Nitric Oxide and Other Endothelium-Derived Factors. Medicina. 2003, 39(4), 333-341.

[14] Delpy, E.; Coste, H.; Gouville, A. C. Effects of Cyclic GMP Elevation on Isoprenaline-Induced Increase in Cyclic AMP and Relaxation in Rat Aortic Smooth Muscle: Role of Phosphodiesterase 3. Br. J. Pharmacol. 1996, 119, 471-8.

[15] Stryer, L. The Molecules of Visual Excitation. Scientific American. 1987, 257, 42-50.

[16] Nyberg-Swenson, B. E. Are Acetylcholine-Induced Acetyl Groups Driving Fuel Cells in the Systems of Transducin, T and G Proteins? Medical Hypothesis. 2002, 58(5), 388-394.

[17] Lehninger, A. L.; Nelson, D. L.; Cox, M. M. Principles of Biochemistry; Worth Publishers: New York, 1993.

[18] Fulton, D.; McGiff, J. C.; Wolin, M. S.; Kaminski, P.; Quilley, J. Evidence Against a Cytochrome P450-Derived Reactive Oxygen Species as the Mediator of the Nitric Oxide-Independent Vasodilator Effect of Bradykinin in the Perfused Heart of the Rat. J. Pharmacol Exp Ther. 1997, 280, 702-709.

[19] Pomposiello, S.; Rhaleb, N. E.; Alva, M.; Carretero, O. A. Reactive Oxygen Species: Role in the Relaxation Induced by Bradykinin or Arachidonic Acid via EDHF in Isolated Porcine Coronary Arteries. J. Cardiovasc Pharmacol. 1999, 34, 567-574.

[20] Lehrer, L. Why Science is Failing us. Wired. Dec. 2011. 